Proceedings of the

International Geometry Center

Vol. 14, no. 2 (2021) pp. 137-153

\title{
Some applications of transversality for infinite dimensional manifolds
}

\author{
Eftekharinasab Kaveh
}

\begin{abstract}
We present some transversality results for a category of Fréchet manifolds, the so-called $M C^{k}$-Fréchet manifolds. In this context, we apply the obtained transversality results to construct the degree of nonlinear Fredholm mappings by virtue of which we prove a rank theorem, an invariance of domain theorem and a version of Borsuk-Ulam type theorem.
\end{abstract}

\begin{abstract}
Анотація. В роботі представлено деякі результати про трансверсальність для категорії $M C^{k}$-многовидів Фреше. Вони застосовуються до побудови степеня нелінійних відображень Фредгольма та доведення з його допомогою теорем про ранг, інваріантність області, а також версії теореми Улама-Борсука.
\end{abstract}

This paper is devoted to the development of transversality and its applications to degree theory of nonlinear Fredholm mappings for non-Banachable Fréchet manifolds. The elaboration is mostly, but not entirely, routine; we shall discuss the related issues.

In attempting to develop transversality and Fredholm theory to Fréchet manifolds we face the following drawbacks which are related to the lack of a suitable topology on a space of continuous linear mappings:

(1) In general, the set of isomorphisms between Fréchet spaces is not open in the space of continuous linear mappings.

(2) In general, the set of Fredholm operators between Fréchet spaces is not open in the space of continuous linear mappings.

2010 Mathematics Subject Classification: 57N75,58B15, 47H11.

Keywords: Transversality, degree of nonlinear Fredholm mappings, Fréchet manifolds

Ключові слова: Трансверсальність, степінь нелінійного відображення Фредгольма, многовид Фреше

DOI: http://dx.doi.org/10.15673/tmgc.v14i2.1939 
Also, a key point in the proof of an infinite dimensional version of Sard's theorem is that a Fredholm mapping $\varphi$ near origin has a local representation of the form $\varphi(u, v)=(u, \eta(u, v))$ for some smooth mapping $\eta$; indeed, this is a consequence of an inverse function theorem.

To obtain a version of Sard's theorem for Fréchet manifolds, based on the ideas of Müller [8], it was proposed by the author ([1]) to consider Fredholm operators which are Lipschitz on their domains. There is an appropriate metrizable topology on a space of Lipschitz linear mappings so that if we employ this space instead of a space of continuous linear mappings, the mentioned openness issues and the problem of stability of Fredholm mappings under small perturbation can be resolved. Furthermore, for mappings belong to a class of differentiability, bounded or $M C^{k}$-differentiability which is introduced in [8], a suitable version of an inverse function theorem is available, [8, Theorem 4.7] and [4, Proposition 7.1].

An example of Lipschitz-Fredholm mapping of class $M C^{k}$ can be found in [6], where the Sard's theorem [1, Theorem 4.3] is applied to classify all the holomorphic functions locally definable; this gives the additional motivation to study further applications of Sard's theorem.

In this paper, we first improve the transversality theorem [2, Theorem 4.2 by considering all mappings of class $M C^{k}$, then use it to prove the parametric transversality theorem. Further, for Lipschitz-Fredholm mappings of class $M C^{k}$ we apply the transversality theorem to construct the degree (due to Cacciappoli, Shvarts and Smale), which is defined as the group of non-oriented cobordism class of $\varphi^{-1}(q)$ for some regular value $q$.

Next, we prove a rank theorem for Lipschitz-Fredholm mappings of class $M C^{k}$, and use it to establish an invariance of domain theorem and a Fredholm alternative theorem. Also, using the parametric transversality theorem we obtain a Borsuk-Ulam type theorem.

\section{Bounded Fréchet MANifolds}

In this section we will briefly recall the basics of $M C^{k}$-Fréchet manifolds for the convenience of readers, which also allows us to establish our notations for the rest of the paper. For more studies, we refer to $[1,2,4]$.

Throughout the paper we assume that $E, F$ are Fréchet spaces and we denote by $C L(E, F)$ the space of all continuous linear mappings from $E$ to $F$ topologized by the compact-open topology.

If $T$ is a topological space by $U \subseteq T$ we mean $U$ is open in $T$.

Let $\varphi: U \subseteq E \rightarrow F$ be a continuous map. If the directional (Gâteaux) derivatives

$$
\mathrm{D} \varphi(x) h=\lim _{t \rightarrow 0} \frac{\varphi(x+t h)-\varphi(x)}{t}
$$


exist for all $x \in U$ and all $h \in E$, and the induced map

$$
\mathrm{D} \varphi(x): U \rightarrow C L(E, F)
$$

is continuous for all $x \in U$, then we say that $\varphi$ is a Keller's differentiable map of class $C_{c}^{1}$. The higher directional derivatives and $C_{c}^{k}$-mappings, $k \geqslant 2$, are defined in the obvious inductive fashion.

To define bounded or $M C^{k}$-differentiability, we endow a Fréchet space $F$ with a translation invariant metric $\varrho$ defining its topology, and then introduce the metric concepts which strongly depend on the choice of $\varrho$. We consider only metrics of the following form

$$
\varrho(x, y)=\sup _{n \in \mathbb{N}} \frac{1}{2^{n}} \frac{\|x-y\|_{F, n}}{1+\|x-y\|_{F, n}},
$$

where $\|\cdot\|_{F, n}$ is a collection of seminorms generating the topology of $F$.

Let $\sigma$ be a metric that defines the topology of a Fréchet space $E$. Let $\mathbb{L}_{\sigma, \varrho}(E, F)$ be the set of all linear mappings $L: E \rightarrow F$ which are (globally) Lipschitz continuous as mappings between metric spaces $E$ and $F$, that is

$$
\mathcal{L i p}(L):=\sup _{x \in E \backslash\left\{0_{F}\right\}} \frac{\varrho\left(L(x), 0_{F}\right)}{\sigma\left(x, 0_{F}\right)}<\infty,
$$

where $\operatorname{Lip}(L)$ is the (minimal) Lipschitz constant of $L$.

The translation invariant metric

$$
\begin{gathered}
\mathbb{d}_{\sigma, \varrho}: \mathbb{L}_{\sigma, \varrho}(E, F) \times \mathbb{L}_{\sigma, \varrho}(E, F) \longrightarrow[0, \infty), \\
(L, H) \mapsto \mathcal{L} i p(L-H)_{\sigma, \varrho},
\end{gathered}
$$

on $\mathbb{L}_{\sigma, \varrho}(E, F)$ turns it into an Abelian topological group. We always topologize the space $\mathbb{L}_{\sigma, \varrho}(E, F)$ by the metric (1.1).

Let $\varphi: U \subseteq E \rightarrow F$ be a continuous map. If $\varphi$ is Keller's differentiable, $\mathrm{D} \varphi(x) \in \mathbb{L}_{\sigma, \varrho}(E, F)$ for all $x \in U$ and the induced map

$$
\mathrm{D} \varphi(x): U \rightarrow \mathbb{L}_{\sigma, \varrho}(E, F)
$$

is continuous, then $\varphi$ is called bounded differentiable or $M C^{1}$ and we write $\varphi^{(1)}=\varphi^{\prime}$. We define for $k>1$ mappings of class $M C^{k}$, recursively.

An $M C^{k}$-Fréchet manifold is a Hausdorff second countable topological space modeled on a Fréchet space with an atlas of coordinate charts such that the coordinate transition functions are all $M C^{k}$-mappings. We define $M C^{k}$-mappings between Fréchet manifolds as usual. Henceforth, we assume that $M$ and $N$ are connected $M C^{k}$-Fréchet manifolds modeled on Fréchet spaces $(F, \varrho)$ and $(E, \sigma)$, respectively.

A mapping $\varphi \in \mathbb{L}_{\sigma, \varrho}(E, F)$ is called Lipschitz-Fredholm operator if its kernel has finite dimension and its image has finite co-dimension. The 
index of $\varphi$ is defined by

$$
\operatorname{Ind} \varphi=\operatorname{dim}(\operatorname{ker} \varphi)-\operatorname{codim}(\operatorname{Img} \varphi) .
$$

We denote by $\mathcal{L} \mathcal{F}(E, F)$ the set of all Lipschitz-Fredholm operators, and by $\mathcal{L F}_{l}(E, F)$ the subset of $\mathcal{L} \mathcal{F}(E, F)$ consisting of those operators of index $l$.

An $M C^{k}$-Lipschitz-Fredholm mapping $\varphi: M \rightarrow N, k \geqslant 1$, is a mapping such that for each $x \in M$ the derivative $\mathrm{D} \varphi(x): T_{x} M \longrightarrow T_{f(x)} N$ is a Lipschitz-Fredholm operator. The index of $\varphi$, denoted by $\operatorname{Ind} \varphi$, is defined to be the index of $\mathrm{D} \varphi(x)$ for some $x$ which does not depend on the choice of $x$, see [1, Definition 3.2 ].

Let $\varphi: M \rightarrow N$ be an $M C^{k}$-mapping, $k \geqslant 1$. We will denote by

$$
T_{x} \varphi: T_{x} M \rightarrow T_{\varphi(x)} N
$$

the tangent map of $f$ at $x \in M$ from the tangent space $T_{x} M$ to the tangent space $T_{\varphi(x)} N$. Say that $\varphi$ is an immersion (resp. submersion) whenever $T_{x} \varphi$ is injective (resp. surjective) and the range $\operatorname{Img}\left(T_{x} \varphi\right)$ (resp. the kernel $\left.\operatorname{ker}\left(T_{x} \varphi\right)\right)$ splits in $T_{\varphi(x)} N$ (resp. $T_{x} M$ ) for any $x \in M$. An injective immersion $f: M \rightarrow N$ which gives an isomorphism onto a submanifold of $N$ is called an embedding. A point $x \in M$ is called a regular point if $\mathrm{D} f(x): T_{x} M \longrightarrow T_{f(x)} N$ is surjective. The corresponding value $f(x)$ is a regular value. Points and values other than regular are called critical points and values, respectively.

Let $\varphi: M \rightarrow N$ be an $M C^{k}$-mapping, $k \geqslant 1$. We say that $\varphi$ is transversal to a submanifold $S \subseteq N$ and write $\varphi \pitchfork S$ if either $\varphi^{-1}(S)=\varnothing$, or if for each $x \in \varphi^{-1}(S)$

(1) $\left(T_{x} \varphi\right)\left(T_{x} M\right)+T_{\varphi(x)} S=T_{\varphi(x)} N$, and

(2) $\left(T_{x} \varphi\right)^{-1}\left(T_{\varphi(x)} S\right)$ splits in $T_{x} M$.

In terms of charts, $\varphi \pitchfork S$ whenever for each $x \in \varphi^{-1}(S)$ there exist charts $(\phi, \mathcal{U})$ around $x$ and $(\psi, \mathcal{V})$ around $\varphi(x)$ such that

$$
\psi: \mathcal{V} \rightarrow \mathcal{V}_{1} \times \mathcal{V}_{2}
$$

is an $M C^{k}$-isomorphism on a product, with

$$
\psi(\varphi(x))=\left(0_{E}, 0_{E}\right) \quad \varphi(S \cap \mathcal{V})=\mathcal{V}_{1} \times\left\{0_{E}\right\} .
$$

Then the composite mapping

$$
\mathcal{U} \stackrel{\varphi}{\rightarrow} \mathcal{V} \stackrel{\psi}{\rightarrow} \mathcal{V}_{1} \times \mathcal{V}_{2} \stackrel{\operatorname{Pr}_{V_{2}}}{\rightarrow} \mathcal{V}_{2}
$$

is an $M C^{k}$-submersion, where $\operatorname{Pr}_{V_{2}}$ is the projection onto $\mathcal{V}_{2}$. 


\section{TRANSVERSALITY THEOREMS}

In this section we generalize [2, Theorem 4.2] and [2, Corollary 4.1] for not necessarily Lipschitz-Fredholm mappings and finite dimensional submanifolds. We will need the following version of the inverse function theorem for $M C^{k}$-mappings.

Theorem 2.1 ([8, Theorem 4.7]). Let $\mathcal{U} \subseteq E, u_{0} \in \mathcal{U}$ and $\varphi: \mathcal{U} \rightarrow E$ an $M C^{k}$-mapping, $k \geqslant 1$. If $\varphi^{\prime}\left(u_{0}\right) \in \operatorname{Aut}(E)$, then there exists $\mathcal{V} \subseteq \mathcal{U}$ of $u_{0}$ such that $\varphi(\mathcal{V})$ is open in $E$ and $\varphi \mid \mathcal{V}: \mathcal{V} \rightarrow \varphi(\mathcal{V})$ is an $M C^{k}$ diffeomorphism.

Proposition 2.2. Let $\varphi: M \rightarrow N$ be an $M C^{k}$-mapping, $S \subset N$ an $M C^{k}$ submanifold and $x \in \varphi^{-1}(S)$. Then $\varphi \pitchfork S$ if and only if there are charts $(\mathcal{U}, \phi)$ around $x$ with $\phi(x)=0_{E}$ and $(\mathcal{V}, \psi)$ around $y=\varphi(x)$ in $S$ with $\psi(y)=0_{F}$ such that the following hold:

(1) There are subspaces $\mathbf{E}_{\mathbf{1}}$ and $\mathbf{E}_{\mathbf{2}}$ of $E$, and $\mathbf{F}_{\mathbf{1}}$ and $\mathbf{F}_{\mathbf{2}}$ of $F$ such that $E=\mathbf{E}_{\mathbf{1}} \oplus \mathbf{E}_{\mathbf{2}}$ and $F=\mathbf{F}_{\mathbf{1}} \oplus \mathbf{F}_{\mathbf{2}}$. Moreover, $\psi(S \cap \mathcal{V})=F_{1}$ and

$$
\begin{aligned}
& \phi(\mathcal{U})=E_{1}+E_{2} \subseteq \mathbf{E}_{\mathbf{1}} \oplus \mathbf{E}_{\mathbf{2}} \\
& \psi(\mathcal{V})=F_{1}+F_{2} \subseteq \mathbf{F}_{\mathbf{1}} \oplus \mathbf{F}_{\mathbf{2}},
\end{aligned}
$$

where $0_{E} \in E_{i} \stackrel{\varrho}{\mathbf{E}_{\mathbf{i}}}$ and $0_{F} \in F_{i} \subseteq \mathbf{F}_{\mathbf{i}}, i=1,2$.

(2) In the charts the local representative of $\varphi$ has the form

$$
\varphi_{\phi \psi}=\bar{\varphi}+\hat{\varphi} \circ \operatorname{Pr}_{E_{2}}
$$

where $\bar{\varphi}: E_{1}+E_{2} \rightarrow F_{1}$ is an $M C^{k}$-mapping, $\hat{\varphi}$ is an $M C^{k}$-isomorphism of $\mathbf{E}_{\mathbf{2}}$ onto $\mathbf{F}_{\mathbf{2}}$ and $\operatorname{Pr}_{E_{2}}: E \rightarrow \mathbf{E}_{\mathbf{2}}$ is the projection.

Proof. Sufficiency. Let $(\mathcal{U}, \phi)$ and $(\mathcal{V}, \psi)$ be charts that satisfy the assumptions we will prove $\varphi \pitchfork S$.

In the charts, by using the identifications $T_{x} M \simeq E, T_{y} \simeq F$, the tangent $\operatorname{map} T_{x} \varphi: T_{x} M \rightarrow T_{y} N$ has the representation

$$
T_{x} \varphi=\varphi_{\phi \psi}^{\prime}\left(0_{E}\right): E \rightarrow F .
$$

Also, we have the identification $T_{y} S \simeq \mathbf{F}_{\mathbf{1}}$.

Let $\operatorname{Pr}_{F_{2}}: F \rightarrow \mathbf{F}_{\mathbf{2}}$ be the projection onto $\mathbf{F}_{\mathbf{2}}$. Since

$$
\varphi_{\phi \psi}^{\prime}\left(0_{E}\right)=(\bar{\varphi})^{\prime}+\hat{\varphi} \circ \operatorname{Pr}_{E_{2}}
$$

and $(\bar{\varphi})^{\prime}(0): E \rightarrow \mathbf{F}_{\mathbf{1}}$, it follows that for all $e \in E$ we have that $e=e_{1}+e_{2} \in$ $\mathbf{E}_{1} \oplus \mathbf{E}_{2}$

$$
\varphi_{\phi \psi}^{\prime}\left(0_{E}\right) e=(\bar{\varphi})^{\prime}\left(0_{E}\right) e+\hat{\varphi} \circ \operatorname{Pr}_{E_{2}}
$$


Thus, $\operatorname{Pr}_{F_{2}} \circ \varphi_{\phi \psi}^{\prime}\left(0_{E}\right) e=\operatorname{Pr}_{F_{2}} \circ \hat{\varphi} \circ \operatorname{Pr}_{F_{2}}(e)$ which means that

$$
\operatorname{Pr}_{F_{2}} \circ \varphi_{\phi \psi}^{\prime}\left(0_{E}\right)=\operatorname{Pr}_{F_{2}} \circ \hat{\varphi} \circ \operatorname{Pr}_{F_{2}}
$$

it is a surjective mapping of $E$ onto $\mathbf{F}_{2}$.

Moreover, we have

$$
\begin{aligned}
\operatorname{ker}\left(\operatorname{Pr}_{F_{2}} \circ \varphi_{\phi \psi}^{\prime}\left(0_{E}\right)\right) & =\varphi_{\phi \psi}^{\prime}\left(0_{E}\right)^{-1}\left(\operatorname{Pr}_{F_{2}}\left(0_{E}\right)\right)=\varphi_{\phi \psi}^{\prime}\left(0_{E}\right)^{-1}\left(F_{1}\right) \\
& =\left\{e=e_{1}+e_{2} \in \mathbf{E}_{\mathbf{1}} \oplus \mathbf{E}_{\mathbf{2}} \mid(\bar{\varphi})^{\prime}\left(0_{E}\right) e+\hat{\varphi}\left(e_{2}\right) \in \mathbf{F}_{1}\right\} \\
& =\left\{e=e_{1}+e_{2} \in \mathbf{E}_{\mathbf{1}} \oplus \mathbf{E}_{\mathbf{2}} \mid \hat{\varphi}\left(e_{2}\right)=0_{F}\right\} \\
& =\left\{e=e_{1}+e_{2} \in \mathbf{E}_{\mathbf{1}} \oplus \mathbf{E}_{\mathbf{2}} \mid e_{2}=0_{E}\right\} \\
& =\mathbf{E}_{1} .
\end{aligned}
$$

Which is an $M C^{k}$-splitting in $E$ with a component $E_{2}$. From (2.2) it follows that

$$
\operatorname{Pr}_{F_{2}} \circ \varphi_{\phi \psi}^{\prime}\left(0_{E}\right)=\hat{\varphi}: \operatorname{Pr}_{E_{2}} \rightarrow \operatorname{Pr}_{F_{2}}
$$

which is an $M C^{k}$-isomorphism.

Necessity. Suppose $\varphi \pitchfork S$. Since $S$ is an $M C^{k}$-submanifold of $N$ and $y=\varphi(x) \in S$, there is a chart $(\mathcal{W}, \mathfrak{w})$ around $y$ having the submanifold property for $S$ in $N$ :

$$
\begin{gathered}
\mathfrak{w}(\mathcal{W})=W_{1}+W_{2} \subset \mathbf{F}_{1} \oplus \mathbf{F}_{2}=F, \\
\mathbb{w}(S \cap \mathcal{W})=W_{1} \subset F, \quad \mathfrak{w}(y)=0_{E} .
\end{gathered}
$$

Also, there is a chart $(\mathcal{X}, \rtimes)$ around $x$ such that $₫(x)=0_{F}, \varphi(\mathcal{X}) \subset \mathcal{W}$ and

$$
\varphi_{\text {xw }}: \mathbb{x}(\mathcal{X}) \cong E \rightarrow \mathfrak{w}(\mathcal{W}) \cong F
$$

is of class $M C^{k}$. It follows that

$$
\varphi_{\text {xw }}\left(0_{E}\right) \circ \operatorname{Pr}_{F_{2}}: E \rightarrow \mathbf{F}_{\mathbf{2}}
$$

is an $M C^{k}$-submersion as $\varphi \pitchfork S$. In other words, $\varphi_{\text {xow }} \circ \operatorname{Pr}_{F_{2}}$ and $\mathbf{E}_{1}:=$ $\varphi_{\text {xwo }}^{\prime}\left(0_{E}\right)^{-1}\left(\mathbf{F}_{\mathbf{1}}\right)$ splits in $E$ with the complement $\mathbf{E}_{\mathbf{2}}$ such that

$$
\eta:=\left.\operatorname{Pr}_{F_{2}} \circ \varphi_{\text {x } \mathrm{v}}\left(0_{E}\right)\right|_{\mathbf{E}_{2}}: \mathbf{E}_{\mathbf{2}} \rightarrow \mathbf{F}_{\mathbf{2}}
$$

is an $M C^{k}$-isomorphism. Consider the following map:

$$
\tau:=\left(\operatorname{Pr}_{E_{1}}+\eta^{-1} \circ \operatorname{Pr}_{F_{2}} \circ \varphi_{\text {x仙 }}\right): \ltimes(\mathcal{X}) \rightarrow \mathfrak{w}(\mathcal{W}) .
$$

It follow that $\tau$ is an $M C^{k}$-mapping, $\tau\left(0_{E}\right)=0_{E}$, and

$$
\tau^{\prime}\left(0_{E}\right)=\left(\operatorname{Pr}_{E_{1}}+\eta^{-1} \circ \operatorname{Pr}_{F_{2}} \circ \varphi_{\text {xw }}\right)^{\prime}\left(0_{E}\right)=\operatorname{Pr}_{E_{1}}+\operatorname{Pr}_{E_{2}}=\operatorname{Id}_{E},
$$

since for all $e=e_{1}+e_{2} \in \mathbf{E}_{\mathbf{1}} \oplus \mathbf{E}_{\mathbf{2}}$ we have that

$$
\left(\varphi_{\text {xow }}\right)^{\prime}\left(0_{E}\right) e=\left(\varphi_{\text {xwo }}\right)^{\prime}\left(0_{E}\right) e_{1}+\left(\varphi_{\text {xw }}\right)^{\prime}\left(0_{E}\right) e_{2} \text {. }
$$


Whence,

$$
\operatorname{Pr}_{F_{2}} \circ\left(\varphi_{\text {șw }}\right)^{\prime}\left(0_{E}\right) e=\operatorname{Pr}_{F_{2}} \circ\left(\varphi_{\text {șw }}\right)^{\prime}\left(0_{E}\right) e_{2}=\tau\left(e_{2}\right)
$$

and therefore

$$
\tau^{-1} \circ \operatorname{Pr}_{F_{2}} \circ\left(\varphi_{\text {șw }}\right)^{\prime}\left(0_{E}\right) e=\tau^{-1}\left(\tau\left(e_{2}\right)\right)=\operatorname{Pr}_{E_{2}} .
$$

By the inverse mapping theorem 2.1, $\tau$ is a local $M C^{k}$-diffeomorphism. Assume $0_{E} \in \mathcal{X}_{1} \subseteq \mathbb{x}(\mathcal{X})$ is small enough. Let also

$$
\aleph_{1}: \mathcal{X}_{1} \rightarrow 0_{E} \in \mathcal{X}_{2} \subseteq E
$$

be an $M C^{k}$-diffeomorphism such that

$$
\tau \circ{x_{1}^{-1}}^{-1} \operatorname{Id}_{F}
$$

Thus,

$$
\operatorname{Pr}_{F_{2}} \circ \tau \circ \varphi_{\text {sow }}=\eta \circ \operatorname{Pr}_{E_{2}} .
$$

If, $e=e_{1}+e_{2} \in \mathbb{x}_{1}\left(\mathcal{X}_{1}\right)$ and $\boldsymbol{x}_{1}^{-1}(e)=\overline{e_{1}}+\overline{e_{2}}$, then by (2.3) and (2.4) we obtain

$$
\begin{aligned}
\tau \circ{\mathrm{x}_{1}^{-1}}^{-1}(e) & =\tau\left(\overline{e_{1}}+\overline{e_{2}}\right) \\
& =\overline{e_{1}}+\eta^{-1} \circ \operatorname{Pr}_{F_{2}} \circ \varphi_{\mathrm{x} \mathrm{w}}\left(\overline{e_{1}}+\overline{e_{2}}\right) \\
& =e_{1}+e_{2} .
\end{aligned}
$$

Therefore, $\overline{e_{1}}=e_{1}$ and $\tau^{-1} \circ \operatorname{Pr}_{F_{2}} \circ \varphi_{\text {xow }}=e_{2}$ and so

$$
\operatorname{Pr}_{F_{2}} \circ \varphi_{\text {xow }}\left(\overline{e_{1}}+\overline{e_{2}}\right)=\tau\left(e_{2}\right)=\tau \circ \operatorname{Pr}_{E_{2}}\left(e_{1}+e_{2}\right) \text {. }
$$

This means that

$$
\operatorname{Pr}_{E_{2}} \circ \varphi_{\text {sow }} \circ \boldsymbol{x}_{1}^{-1}(e)=\eta \circ \operatorname{Pr}_{E_{2}}(e)
$$

for all $e \in \mathbb{x}_{1}\left(\mathcal{X}_{1}\right)$.

Now, define

$$
\begin{array}{ll}
\phi:=\boldsymbol{x}_{1} \circ \boldsymbol{x}, & \mathcal{U}:=x^{-1}(\mathcal{X}), \\
\psi:=w, & \mathcal{V}:=\text { small enough neighborhoodof } y \text { in } \mathcal{W} .
\end{array}
$$

Then, $(\phi, \mathcal{U})$ and $(\psi, \mathcal{V})$ are the desired charts. Indeed,

$$
\begin{aligned}
\varphi_{\phi \psi} & =\mathbb{w} \circ \varphi \circ\left(\mathbb{x}_{1} \circ \mathbb{x}\right) \\
& =\mathbb{w} \circ \varphi \circ \mathbb{x}^{-1} \circ \mathbb{x}^{-1}=\varphi_{\mathbb{x} \mathfrak{w}} \circ \mathbb{x}_{1}^{-1} .
\end{aligned}
$$

Thus,

$$
\begin{aligned}
\varphi_{\phi \psi} & =\operatorname{Pr}_{F_{1}} \circ \varphi_{\text {sov }} \circ \mathbb{x}_{1}^{-1}+\operatorname{Pr}_{F_{2}} \circ \varphi_{\text {sow }} \circ \mathbb{x}_{1}^{-1} \\
& =\bar{\varphi}+\hat{\varphi} \circ \operatorname{Pr}_{E_{2}},
\end{aligned}
$$

whenever we set $\hat{\varphi}:=\eta$ and $\bar{\varphi}:=\operatorname{Pr}_{E_{1}} \circ \varphi_{\text {xw }} \circ x_{1}^{-1}$. 
Theorem 2.3 (Transversality Theorem). Let $\varphi: M \rightarrow N$ be an $M C^{k}$ mapping, $k \geqslant 1, S \subset N$ an $M C^{k}$-submanifold, and $\varphi \pitchfork S$. Then $\varphi^{-1}(S)$ is either empty of $M C^{k}$-submanifold of $M$ with

$$
\left(T_{x} \varphi\right)^{-1}\left(T_{y} S\right)=T_{x}\left(\varphi^{-1}(S)\right), x \in \varphi^{-1}(S), y=\varphi(x) .
$$

If $S$ has finite co-dimension in $N$, then $\operatorname{codim}\left(\varphi^{-1}(S)\right)=\operatorname{codim} S$. Moreover, if $\operatorname{dim} S=m<\infty$ and $\varphi$ is an $M C^{k}$-Lipschitz-Fredholm mapping of index $l$, then $\operatorname{dim} \varphi^{-1}(S)=l+m$.

Proof. Let $x \in \varphi^{-1}(S)$. Then by Proposition 2.2 there are charts $(\phi, \mathcal{U})$ around $x$ and $(\psi, \mathcal{V})$ around $y=\varphi(x)$ such that

$$
\begin{gathered}
\phi(\mathcal{U})=E_{1}+E_{2} \subseteq \mathbf{E}_{\mathbf{1}} \oplus \mathbf{E}_{\mathbf{2}}, \\
\psi(\mathcal{V})=F_{1}+F_{2} \subseteq \mathbf{F}_{\mathbf{1}} \oplus \mathbf{F}_{\mathbf{2}}, \\
\varphi_{\phi \psi}=\bar{\varphi}+\hat{\varphi} \circ \operatorname{Pr}_{E_{2}},
\end{gathered}
$$

where $\bar{\varphi}: E_{1}+E_{2} \rightarrow F_{1}$ is an $M C^{k}$-mapping, $\hat{\varphi}$ is an $M C^{k}$-isomorphism of $\mathbf{E}_{2}$ onto $\mathbf{F}_{2}$, and $\operatorname{Pr}_{E_{2}}: E \rightarrow \mathbf{E}_{2}$ is the projection.

Let $\hat{e} \in \varphi^{-1}(S) \cap \mathcal{U}$. Then $\hat{f}=\varphi(\hat{e}) \in S \cap \mathcal{V}$ and $\psi(\varphi(\hat{e})) \in F_{1} \subset \mathbf{F}_{1}$. Due to (2.5), if $\phi(\hat{e})=e_{1}+e_{2} \in E_{1}+E_{2}$, then

$$
\begin{aligned}
\varphi_{\phi \psi}(\phi(\hat{e})) & =\varphi_{\phi \psi}\left(e_{1}+e_{2}\right) \\
& =\bar{\varphi}\left(e_{1}+e_{2}\right)+\hat{\varphi}\left(e_{2}\right) \in F_{1} \subset \mathbf{F}_{\mathbf{1}} .
\end{aligned}
$$

Therefore $\hat{\varphi}\left(e_{2}\right)=0_{E}$ and $e_{2}=0_{E}$, since $\hat{\varphi}\left(e_{2}\right) \in \mathbf{F}_{\mathbf{2}}$ and $\mathbf{F}_{\mathbf{1}} \cap \mathbf{F}_{\mathbf{2}}=\left\{0_{F}\right\}$.

Thus, $\phi(\hat{e}) \in F_{1}$ for all $\hat{e} \in \varphi^{-1}(S) \cap \mathcal{U}$. Hence,

$$
E_{1} \subset \phi\left(\varphi^{-1}(S) \cap \mathcal{U}\right),
$$

since for each $e_{1} \in E_{1}$ we have that

$$
\varphi_{\phi \psi}\left(e_{1}\right)=\bar{\varphi}\left(e_{1}\right)+\hat{\varphi} \circ \operatorname{Pr}_{E_{2}}\left(e_{1}\right)=\bar{\varphi}\left(e_{1}\right) \in F_{1} .
$$

Hence, $\psi \circ \varphi \circ \phi^{-1}\left(e_{1}\right) \in F_{1}$ implies that

$$
\varphi \circ \phi^{-1}\left(e_{1}\right) \in \psi^{-1}\left(F_{1}\right)=S \cap \mathcal{V}
$$

and so $\varphi \circ \phi^{-1}\left(e_{1}\right) \in S$ which means $\phi^{-1}\left(e_{1}\right) \in \varphi^{-1}(S) \cap \mathcal{V}$ and that yields $e_{1} \in \phi\left(\varphi^{-1}(S) \cap \mathcal{V}\right)$. Therefore, for each $x \in \varphi^{-1}(S)$ there is a chart $(\phi, \mathcal{U})$ with

$$
\phi(\mathcal{U})=E_{1}+E_{2} \subset \mathbf{E}_{1} \oplus \mathbf{E}_{2},
$$

$\phi(x)=0_{E}$, and $\phi\left(\varphi^{-1}(S) \cap \mathcal{V}\right)=E_{1}$, which means that $\varphi^{-1}(S)$ is an $M C^{k}$-submanifold in $M$.

In the charts, we have that

$$
T_{x} \simeq E, \quad T_{y} N \simeq F, \quad T_{x}\left(\varphi^{-1}(S)\right) \simeq \mathbf{E}_{\mathbf{1}}, \quad T_{y} S \simeq \mathbf{F}_{\mathbf{1}} .
$$


Then from the proof of Proposition 2.2 we also get that

$$
\varphi_{\phi \psi}^{\prime}\left(0_{E}\right)^{-1}\left(\mathbf{F}_{\mathbf{1}}\right)=\mathbf{E}_{\mathbf{1}}
$$

which yields $\left(T_{x} \varphi\right)^{-1}\left(T_{y} S\right)=T_{x}\left(\varphi^{-1}(S)\right)$.

If $S$ has finite co-dimension then $\mathbf{F}_{\mathbf{2}}$ has finite dimension and thus by Proposition 2.2,

$$
\operatorname{codim}\left(\varphi^{-1}(S)\right)=\operatorname{codim}\left(\varphi^{-1}(S \cap \mathcal{V})\right)=\operatorname{dim}\left(\mathbf{F}_{2}\right)=\operatorname{codim}(S) .
$$

The proof of the last statement is standard.

As an immediate consequence we obtain:

Corollary 2.4. Let $\varphi: M \rightarrow N$ be an $M C^{k}$-mapping, $k \geqslant 1$. If $q$ is a regular value of $\varphi$, then the level set $\varphi^{-1}(q)$ is a submanifold of $M$ and its tangent space at $p=\varphi(q)$ is $\operatorname{ker} T_{p} \varphi$. Moreover, if $q$ is a regular value of $\varphi$ and $\varphi$ is an $M C^{k}$-Lipschitz-Fredholm mapping of index $l$, then $\operatorname{dim}\left(\varphi^{-1}(S)\right)=l$.

To prove the parametric transversality theorem we apply the following Sard's theorem.

Theorem 2.5 ([2, Theorem 3.2]). If $\varphi: M \rightarrow N$ is an $M C^{k}$-LipschitzFredholm map with $k>\max \{\operatorname{Ind} \varphi, 0\}$. Then, the set of regular values of $\varphi$ is residual in $N$.

Theorem 2.6 (The Parametric Transversality Theorem). Let $A$ be a manifold of dimension $n, S \subset N$ a submanifold of finite co-dimension $m$. Let also $\varphi: M \times A \rightarrow N$ be an $M C^{k}$-mapping, $k \geqslant\{1, n-m\}$. If $\varphi \pitchfork S$, then the set of all points $x \in M$ such that the mappings

$$
\varphi_{x}: A \rightarrow N,\left(\varphi_{x}(\cdot):=\varphi(x, \cdot)\right)
$$

are transversal to $S$, is residual in $M$.

Proof. Let $\mathbf{S}=\varphi^{-1}(S), \operatorname{Pr}_{M}: M \times A \rightarrow M$ the projection onto $M$ and $\operatorname{Pr}_{\mathbf{S}}$ be its restriction to $\mathbf{S}$. First we prove that $\operatorname{Pr}_{\mathbf{S}}$ is an $M C^{k}$-FredholmLipschitz mapping of index $n-m$, i.e.,

$$
T_{(m, a)} \operatorname{Pr}_{\mathbf{S}}: T_{(m, a)} \mathbf{S} \rightarrow T_{m} M
$$

is a Lipschitz-Fredholm operator of index $n-m$.

By Theorem 2.3 the inverse image $\mathbf{S}$ is an $M C^{k}$-submanifold of $M \times A$, with model space $\mathbb{S}$, so that $\operatorname{Pr}_{\mathbf{S}}$ is an $M C^{k}$-mapping.

Let $\pi_{M}$ and $\pi_{\mathbf{S}}$ be the local representatives of $\operatorname{Pr}_{M}$ and $\operatorname{Pr}_{\mathbf{S}}$, respectively. We show that $\pi_{M}$ and consequently $\pi_{\mathbf{S}}$ are Lipschitz-Fredholm operators of 
index $n-m$. Finite dimensionality of $\mathbb{R}^{n}$ and closedness of $\mathbb{S}$ implies that

$$
K:=\mathbb{S}+\left(\{0\} \times \mathbb{R}^{n}\right)
$$

is closed in $E \times \mathbb{R}^{n}$. Also, codim $K$ is finite because it contains the finite codimensional subspace $\mathbb{S}$. Therefore $K$ has a finite-dimensional complement $K_{1} \subset E \times\{0\}$, that is $E \times \mathbb{R}^{n}=K \oplus K_{1}$. Let $K_{2}:=\mathbb{S} \cap\{0\} \times \mathbb{R}^{n}$. Since $K_{2} \subset \mathbb{R}^{n}$ we can choose closed subspaces $\mathbb{S}_{1} \subset \mathbb{R}^{n}$ and $\mathbb{R}_{0} \subset\{0\} \times \mathbb{R}^{n}$ such that $\mathbb{S}=\mathbb{S}_{1} \oplus K_{1}$ and $\{0\} \times \mathbb{R}^{n}=K_{1} \oplus \mathbb{R}_{0}$. Whence,

$$
K=\mathbb{S}_{1} \oplus K_{1} \oplus \mathbb{R}_{0}, \quad E \times \mathbb{R}^{n}=\mathbb{S}_{1} \oplus K_{1} \oplus \mathbb{R}_{0} \oplus K_{2} .
$$

The mapping $\left.\pi_{\mathbf{S}}\right|_{\mathbb{S}_{1} \oplus K_{2}}: \mathbb{S}_{1} \oplus K_{2} \rightarrow E$ is an isomorphism, $K_{1}=$ ker $\pi_{\mathbf{S}}$, and $\pi_{M}\left(K_{2}\right)$ is a finite dimensional complement to $\pi_{M}(\mathbb{S})$ in $\mathbb{R}^{n}$. Thus, $\pi_{M}$ is a Lipschitz-Fredholm operator and we have that

$$
\begin{aligned}
\operatorname{Ind} \pi_{M} & =\operatorname{dim} K_{1}-\operatorname{dim} K_{2} \\
& =\operatorname{dim}\left(K_{1} \oplus \mathbb{R}_{0}\right)-\operatorname{dim}\left(\mathbb{R}_{0} \oplus K_{2}\right) .
\end{aligned}
$$

Since, $K_{1} \oplus \mathbb{R}_{0}=\{0\} \times \mathbb{R}^{n}$ and $\mathbb{R}_{0} \oplus K_{2}$ is a complement to $\mathbb{S}$ in $E \times \mathbb{R}^{n}$ and therefore its dimension is $n$, so the index of $\pi_{M}$ is $n-m$.

Now, we prove that if $x$ is a regular value of $\operatorname{Pr}_{\mathbf{S}}$ if and only if $\varphi_{x} \pitchfork S$. From the definition of $\varphi \pitchfork$ we have $\forall(x, a) \in \mathbf{S}$

$$
\left(T_{(x, a)} \varphi\right)\left(T_{x} M \times T_{a} A\right)+T_{\varphi(x, a)} S=T_{\varphi(x, a)} N,
$$

and

$$
\left(T_{(x, a)} \varphi\right)^{-1}\left(T_{\varphi_{(x, a)}} S\right) \text { splits in } T_{x} M \times T_{a} A .
$$

Since $A$ has finite dimension, it follows that the mapping $a \in A \mapsto \varphi(x, a)$ for a fixed $x \in M$ is transversal to $S$ if and only if

$$
\forall(x, a) \in \mathbf{S}, T_{a} \varphi_{x}\left(T_{a} A\right)+T_{\varphi(x, a)} S=T_{\varphi(x, a)} S .
$$

Since $\operatorname{Pr}_{\mathbf{S}}$ is a Lipschitz-Fredholm mapping, $\operatorname{ker} T \operatorname{Pr}_{\mathbf{S}}$ splits at any point as its dimension is finite. Hence $x$ is a regular value of $\operatorname{Pr}_{\mathbf{S}}$ if and only if

$$
\forall(x, a) \in \mathbf{S}, \forall v \in T_{x} M, \exists u \in T_{a} A: T_{(v, u)} \varphi(v, u) \in T_{(x, a)} S .
$$

Pick $x \in M$ and $a \in A$ such that $(x, a) \in \mathbf{S}$ and let $w \in T_{(x, a)} S$. By (2.6) and (2.7) we obtain that there exist $v \in T_{a} A, x_{1} \in T_{x} M, y_{1} \in T_{(x, a)} S$ such that

$$
T_{(x, a)} \varphi\left(v, x_{1}\right)+y_{1}=w .
$$

Then, there exists $x_{2} \in T_{x} M$ such that $T_{(x, a)} \varphi\left(v, x_{2}\right) \in T_{\varphi_{(x, a)}} S$. Hence,

$$
\begin{aligned}
w & =T_{(x, a)} \varphi\left(v, x_{1}\right)-T_{(x, a)} \varphi\left(v, x_{2}\right)+T_{(x, a)} \varphi\left(v, x_{2}\right)+y_{1} \\
& =T_{(x, a)} \varphi\left(0, x_{1}-x_{2}\right)+T_{(x, a)} \varphi\left(v, x_{2}\right)+y_{1} \\
& =T_{(x, a)} \varphi(0, u)+T_{\varphi(x, a)} S+y_{2} \in T_{a} \varphi_{x}\left(T_{a} A\right),
\end{aligned}
$$

where $u=x_{1}-x_{2}$ and $y_{2}=T_{(x, a)} \varphi\left(v, x_{2}\right)+y_{1} \in T_{\varphi_{(x, a)}} S$. Thus, (2.8) holds. 
Now we show that (2.8) implies (2.9). Pick $a \in A, x \in M$ such that $(x, a) \in \mathbf{S}$. Let $v \in T_{x} M, a_{1} \in T_{a} A, y_{1} \in T_{\varphi_{(x, a)}} S$ and set

$$
w:=T_{(x, a)} \varphi_{\left(v, x_{1}\right)}+y_{1} .
$$

By (2.8) there exist $a_{2} \in T_{a} A$ and $y_{2} \in T_{\varphi_{(x, a)}} S$ such that

$$
w=T_{a} \varphi_{x}\left(a_{2}\right)+y_{2} \text {. }
$$

Then,

$$
\begin{aligned}
0_{E} & =T_{(x, a)} \varphi\left(v, a_{1}\right)-T_{a} \varphi_{x}\left(a_{2}\right)+y_{1}-y_{2} \\
& =T_{(x, a)} \varphi\left(v, a_{1}-a_{2}\right)+y_{1}-y_{2},
\end{aligned}
$$

SO

$$
T_{(x, a)} \varphi\left(v, a_{1}-a_{2}\right)=y_{2}-y_{1} \in T_{\varphi_{(x, a)}} S,
$$

and thus (2.9) holds. Thus, we showed that if $x$ is a regular value of $\operatorname{Pr}_{\mathbf{S}}$ if and only if $\varphi_{x} \pitchfork S$. Since $\operatorname{Pr}_{\mathbf{S}}: \mathbf{S} \rightarrow M$ is a Lipschitz-Fredholm of class $M C^{k}$ with the index $n-m$ and

$$
\operatorname{codim} \mathbf{S}=\operatorname{codim} S=m
$$

and $k>\{0, n-m\}$, the Sard's theorem 2.5 concludes the theorem.

\section{The Degree of Lipschitz-Fredholm MAPpings}

In this section we construct the degree of $M C^{k}$-Lipschitz-Fredholm mappings and apply it to prove an invariance of domain theorem, a rank theorem and a Borsuk-Ulam type theorem. The construction of the degree relies on the following transversality result.

Theorem 3.1 ([2, Theorem 3.3]). Let $\varphi: M \rightarrow N$ be an $M C^{k}$-LipschitzFredholm mapping, $k \geqslant 1$. Let also $\imath: \mathcal{A} \rightarrow N$ be an $M C^{1}$-embedding of a finite dimension manifold $\mathcal{A}$ with $k>\max \{\operatorname{Ind} \varphi+\operatorname{dim} \mathcal{A}, 0\}$. Then there exists an $M C^{1}$ fine approximation $\mathbf{g}$ of $\imath$ such that $\mathbf{g}$ is embedding and $\varphi \mathbf{i} \mathbf{g}$. Moreover, suppose $S$ is a closed subset of $\mathcal{A}$ and $\varphi \pitchfork \imath(S)$, then $\mathbf{g}$ can be chosen so that $\imath=\mathbf{g}$ on $S$.

We shall need the following theorem that gives the connection between proper and closed mappings.

Theorem 3.2 ([9, Theorem 1.1]). Let A, B be Hausdorff manifolds, where $A$ is a connected infinite dimensional Fréchet manifold, and $B$ satisfies the first countability axiom, and let $\varphi: A \rightarrow B$ be a continuous closed non-constant map. Then $\varphi$ is proper. 
Let $\varphi: M \rightarrow N$ be a non-constant closed Lipschitz-Fredholm mapping with index $l \geqslant 0$ of class $M C^{k}$ such that $k>l+1$. If $q$ is a regular value of $\varphi$, then by Theorem 3.2 and Corollary 2.4 the preimage $\varphi^{-1}(q)$ is a compact submanifold of dimension $l$.

Let $\imath:[0,1] \hookrightarrow N$ be an $M C^{1}$-embedding that connects two distinct regular values $q_{1}$ and $q_{2}$. By Theorem 3.1 we may suppose $\imath$ is transversal to $\varphi$. Thus, by Theorem 2.3 the preimage $\mathbf{M}:=\varphi^{-1}(\imath([0,1]))$ is a compact $(l+1)$-dimensional submanifold of $M$ such that its boundary, $\partial \mathbf{M}$, is the disjoint union of $\varphi^{-1}\left(q_{1}\right)$ and $\varphi^{-1}\left(q_{2}\right), \partial \mathbf{M}=\varphi^{-1}\left(q_{1}\right) \amalg \varphi^{-1}\left(q_{2}\right)$. Therefore, $\varphi^{-1}\left(q_{1}\right)$ and $\varphi^{-1}\left(q_{2}\right)$ are non-oriented cobordant which gives the invariance of the mapping. Following Smale [10] we associate to $\varphi$ a degree, denoted by $\operatorname{deg} \varphi$, defined as the non-oriented cobordism class of $\varphi^{-1}(q)$ for some regular value $q$. If $l=0$, then $\operatorname{deg} \varphi \in \mathbb{Z}_{2}$ is the number modulo 2 of preimage of a regular value.

Let $\mathcal{O} \cong M$. Suppose $\varphi: \overline{\mathcal{O}} \rightarrow N$ is a non-constant closed continuous mapping such that its restriction to $\mathcal{O}$ is an $M C^{k+1}$-Lipschitz-Fredholm mapping of index $k, k \geqslant 0$. Let $p \in N \backslash \varphi(\partial \overline{\mathcal{O}})$ and let $\mathbf{p}$ a regular value of $\varphi$ in the connected component of $N \backslash \varphi(\partial \overline{\mathcal{O}})$ containing $p$, the existence of such regular value follows from Sard's theorem 2.5. Again, we associate to $\varphi$ a degree, $\operatorname{deg}(\varphi, p)$, defined as non-oriented class of $k$-dimensional compact manifold $\varphi^{-1}(\mathbf{p})$. This degree does not depend on the choice of $\mathbf{p}$.

The following theorem which presents the local representation of $M C^{k_{-}}$ mappings is crucial for the rest of the paper.

Theorem 3.3 ([1, Theorem 4.2]). Let $\varphi: \mathcal{U} \cong E \rightarrow F$ be an $M C^{k}$ mapping, $k \geqslant 1, u_{0} \in \mathcal{U}$. Suppose that $\mathrm{D} \varphi\left(u_{0}\right)$ has closed split image $\mathbf{F}_{\mathbf{1}}$ with closed topological complement $\mathbf{F}_{\mathbf{2}}$ and split kernel $\mathbf{E}_{\mathbf{2}}$ with closed topological complement $\mathbf{E}_{1}$. Then, there are two open sets $\mathcal{U}_{1} \subseteq \mathcal{U}$ and $\mathcal{V} \cong \mathbf{F}_{\mathbf{1}} \oplus \mathbf{E}_{\mathbf{2}}$ and an $M C^{k}$-diffeomorphism $\Psi: \mathcal{V} \rightarrow \mathcal{U}_{1}$, such that

$$
(\varphi \circ \Psi)(f, e)=(f, \eta(f, e))
$$

for all $(f, e) \in \mathcal{V}$, where $\eta: \mathcal{V} \rightarrow \mathbf{E}_{\mathbf{2}}$ is an $M C^{k}$-mapping.

Theorem 3.4 (Rank theorem for $M C^{k}$-mappings). Let $\varphi: \mathcal{U} \subseteq E \rightarrow F$ be an $M C^{k}$-mapping, $k \geqslant 1$. Suppose $u_{0} \in \mathcal{U}$ and $\mathrm{D} \varphi\left(u_{0}\right)$ has closed split image $\mathbf{F}_{\mathbf{1}}$ with closed complement $\mathbf{F}_{\mathbf{2}}$ and split kernel $\mathbf{E}_{\mathbf{2}}$ with closed complement $\mathbf{E}_{\mathbf{1}}$. Also, assume

$$
\left.\mathrm{D} \varphi(u)\right|_{\mathbf{E}_{1}}: \mathbf{E}_{1} \rightarrow \mathrm{D} \varphi(u)(E)
$$

is an $M C^{k}$-isomorphism for each $u \in \mathcal{U}$ and $\mathrm{D} \varphi(\mathcal{U})(E)$ is closed in $F$. Then, there exist open sets

$$
\mathcal{U}_{1} \cong \mathbf{F}_{1} \oplus \mathbf{E}_{2}, \quad \mathcal{U}_{2} \cong E, \mathcal{V}_{1} \cong F, \mathcal{V}_{2} \cong F,
$$


and $M C^{k}$-diffeomorphisms $\phi: \mathcal{V}_{1} \rightarrow \mathcal{V}_{2}$ and $\psi: \mathcal{U}_{1} \rightarrow \mathcal{U}_{2}$ such that

$$
(\phi \circ \varphi \circ \psi)(f, e)=(f, 0), \quad \forall(f, e) \in \mathcal{U}_{1} .
$$

Proof. By Theorem 3.3 there exits an $M C^{k}$-diffeomorphism

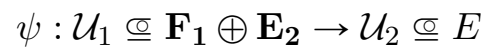

such that

$$
\varphi(f, e)=(\varphi \circ \psi)(f, e)=(f, \eta(f, e)),
$$

where $\eta: \mathcal{V} \rightarrow \mathbf{E}_{\mathbf{2}}$ is an $M C^{k}$-mapping. Let $\operatorname{Pr}_{1}: F \rightarrow \mathbf{F}_{1}$ be the projection. Then $\operatorname{Pr}_{1} \circ \mathrm{D} \varphi(f, e)(w, v)=(w, 0)$, for $w \in \mathbf{F}_{\mathbf{1}}$ and $v \in \mathbf{E}_{\mathbf{2}}$ since

$$
\mathrm{D} \varphi(f, e)(w, v)=(w, \mathrm{D} \eta(f, e)(w, v)) .
$$

Hence, $\left.\operatorname{Pr}_{1} \circ \mathrm{D} \varphi(f, e)\right|_{\mathbf{F}_{\mathbf{1}} \times\{0\}}$ is the identity mapping, $\operatorname{Id}_{\mathbf{F}_{\mathbf{1}}}$, on $\mathbf{F}_{\mathbf{1}}$. Thereby,

$$
\left.\mathrm{D} \varphi(f, e)\right|_{\mathbf{F}_{\mathbf{1}} \times\{0\}}: \mathbf{F}_{\mathbf{1}} \times\{0\} \rightarrow \mathrm{D} \varphi(f, e)\left(\mathbf{F}_{\mathbf{1}} \oplus \mathbf{E}_{\mathbf{2}}\right)
$$

is one-to-one and therefore by our assumption

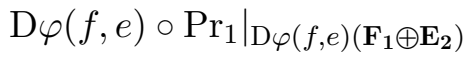

is the identity mapping. Suppose

$$
(w, \mathrm{D} \eta(f, e)(w, v)) \in \mathrm{D} \varphi(f, e)\left(\mathbf{F}_{\mathbf{1}} \oplus \mathbf{E}_{\mathbf{2}}\right) .
$$

Then we obtain $\operatorname{D} \eta(f, e) v=0$ for all $v \in \mathbf{E}_{\mathbf{2}}$, which means that $\mathrm{D}_{2} \eta(f, e)=$ 0 , since

$$
\begin{aligned}
\left(\mathrm{D} \varphi(f, e) \circ \operatorname{Pr}_{1}\right)(w, \mathrm{D} \eta(f, e)(w, v)) & =\mathrm{D} \varphi(f, e)(w, 0) \\
& =(w, \mathrm{D} \eta(f, e)(w, 0)) \\
& =\left(w, \mathrm{D}_{1}(f, e) w\right) .
\end{aligned}
$$

Therefore

$$
\mathrm{D}^{2} \varphi(f, e) v=\left(0, \mathrm{D}_{2} \eta(f, e) v\right),
$$

i.e., $\mathrm{D}^{2} \varphi(f, e)=0$ which means that $\varphi$ does not depend on the variable $y \in \mathbf{E}_{\mathbf{2}}$. Let $\operatorname{Pr}_{2}: \mathbf{F}_{\mathbf{1}} \oplus \mathbf{E}_{\mathbf{2}} \rightarrow \mathbf{F}_{\mathbf{1}}$ be the projection and

$$
\varphi_{f}:=\varphi(f, e)=\left(\varphi \circ \operatorname{Pr}_{2}\right)(f, e) \text {, }
$$

so that

$$
\varphi_{f}: \operatorname{Pr}_{2}\left(\mathcal{U}_{1}\right) \subset \mathbf{F}_{\mathbf{1}} \rightarrow F .
$$

Let also $\mathbf{v}_{\mathbf{0}}:=\left(\operatorname{Pr}_{2} \circ \psi^{-1}\right)\left(u_{0}\right)$ and $\mathcal{V} \subseteq \mathcal{U}$ be an open neighborhood of $\mathbf{v}_{\mathbf{0}}$. Define the following mapping

$$
\Phi: \mathcal{V} \times \mathbf{F}_{\mathbf{2}} \rightarrow \mathbf{F}_{\mathbf{1}} \oplus \mathbf{F}_{\mathbf{2}}, \quad \Phi(f, e)=\varphi(f)+(0, e) .
$$

By the open mapping theorem

$$
\mathrm{D} \Phi\left(\mathbf{v}_{\mathbf{0}}, 0\right)=\left(\mathrm{D} \varphi\left(\mathbf{v}_{\mathbf{0}}\right), \operatorname{Id}_{\mathbf{F}_{2}}\right): E \oplus \mathbf{F}_{\mathbf{2}} \rightarrow F
$$


is a linear $M C^{k}$-isomorphism, where $\operatorname{Id}_{\mathbf{F}_{2}}$ is the identity mapping of $\mathbf{F}_{\mathbf{2}}$. Now $\Phi$ satisfies the inverse function theorem 2.1 at $\mathbf{v}_{0}$, whence there exist $\mathcal{V}_{1}, \mathcal{V}_{2} \subseteq F$ such that $\left(\mathbf{v}_{\mathbf{0}}, 0\right) \in \mathcal{V}_{2}$ and $\Phi\left(\mathbf{v}_{\mathbf{0}}, 0\right)=\varphi_{\mathbf{v}_{\mathbf{0}}}\left(\mathbf{v}_{\mathbf{0}}\right) \in \mathcal{V}_{1}$ and an $M C^{k}-$ diffeomorphism $\phi: \mathcal{V}_{1} \rightarrow \mathcal{V}_{2}$ such that $\phi^{-1}=\left.\Phi\right|_{\mathcal{V}_{1}}$. Thus, for $(f, 0) \in \mathcal{V}_{2}$ we have

and therefore,

$$
(\phi \circ \varphi)(f)=(\varphi \circ \Phi)(f, 0)=(f, 0),
$$

$$
(\phi \circ \varphi \circ \psi)(f, e)=(f, 0), \quad \forall(f, e) \in \mathcal{U}_{1} .
$$

As an immediate consequence we get the following:

Corollary 3.5 (Rank theorem for Lipschitz-Fredholm mappings). Let

$$
\varphi: M \rightarrow N
$$

be an $M C^{\infty}$-Lipschitz-Fredholm mapping of index $k$ and

$$
\operatorname{dim} \operatorname{ker} \mathrm{D} \varphi(x)=m, \quad \forall x \in M .
$$

Let $\complement_{1}$ and $\complement_{2}$ be the topological complements of $\mathbb{R}^{m}$ in $E$ and $\mathbb{R}^{m-k}$ in $F$, respectively. Then, there exist charts

$$
\begin{gathered}
\phi: \mathcal{U} \subseteq M \rightarrow E=\mathbb{R}^{m} \oplus \complement_{1} \text { with } \phi(x)=0_{E}, \\
\psi: \mathcal{V} \subseteq N \rightarrow F=\mathbb{R}^{m-k} \oplus \complement_{2} \text { with } \quad \psi(x)=0_{F},
\end{gathered}
$$

such that

$$
\psi \circ \varphi \circ \phi^{-1}(f, 0)=(f, 0) .
$$

A version of a constant rank theorem for mappings from manifolds modeled on locally convex spaces to finite dimensional manifolds is obtained in [5]. Also, in the case of Banach manifolds a constant rank theorem can be found in [7] and [11].

The following theorem gives the openness property of the set of LipschitzFredholm mappings.

Theorem 3.6 ([1, Theorem 3.2]). The set $\mathcal{L F}(E, F)$ is open in $\mathcal{L}_{d, g}(E, F)$ with respect to the topology defined by the metric (1.1). Furthermore, the function $T \rightarrow \operatorname{Ind} T$ is continuous on $\mathcal{L F}(E, F)$, and hence it is constant on connected components of $\mathcal{L} \mathcal{F}(E, F)$.

The proof of the following theorem is a modification of [11, Theorem 2].

Theorem 3.7. Let $\varphi: M \rightarrow N$ be a Lipschitz-Fredholm mapping of class $M C^{k}, k \geqslant 1$. Then the set

$$
\operatorname{Sing}(\varphi):=\{m \mid \mathrm{D} \varphi(m) \text { is not injective }\}
$$

is nowhere dense in $M$. 
Proof. This is a local problem so we assume $M$ is an open set in $E$ and $N$ is an open set in $F$. Let $s \in \operatorname{Sing}(\varphi)$ be arbitrary and $\mathcal{U}$ an open neighborhood of $s$ in $\operatorname{Sing}(\varphi)$. For each $n \in \mathbb{N} \cup\{0\}$ define

$$
S_{n}:=\{m \in M \mid \operatorname{dim} \mathrm{D} \varphi(m) \geqslant n\} .
$$

Then $M=M_{0} \supset M_{1} \supset \cdots$, whence there exists a unique $n_{0}$ such that

$$
M=M_{n_{0}} \neq M_{n_{0}+1} .
$$

Let $m_{0} \in M_{n_{0}} \backslash M_{n_{0}+1}$ be such that $\operatorname{dim} \operatorname{ker} \mathrm{D} \varphi\left(m_{0}\right)=n_{0}$. By Theorem 3.6, there exists an open neighborhood $\mathcal{V}$ of $m_{0}$ in $\mathcal{U}$ such that for all $v \in \mathcal{V}$ we have that $\operatorname{dim} \operatorname{ker} \mathrm{D} \varphi(v) \leqslant n_{0}$ and hence $\operatorname{dim} \mathrm{D} \varphi(v)=n_{0} \geqslant 1$. By Corollary 3.5, there is a local representative $\varphi$ around zero such that

$$
\psi \circ \varphi \circ \phi^{-1}(f, e)=(f, 0)
$$

for $(f, e) \in \complement_{1} \oplus \mathbb{R}^{n_{0}}$, which contradicts the injectivity of $\varphi$. Therefore, $\operatorname{Sing}(\varphi)$ contains a nonempty open set. The closedness of $\operatorname{Sing}(\varphi)$ is obvious in virtue of Theorem 3.6.

Theorem 3.8 (Invariance of domain for Lipschitz-Fredholm mappings). Let $\varphi: M \rightarrow N$ be an $M C^{k}$-Lipschitz-Fredholm mapping of index zero, $k>1$. If $\varphi$ is locally injective, then $\varphi$ is open.

Proof. Let $p \in U \subseteq M$ and $q=\varphi(p)$. The point $p$ has a connected open neighborhood $\mathcal{U} \cong M$ such that $\left.\varphi\right|_{\overline{\mathcal{U}}}: \overline{\mathcal{U}} \rightarrow N$ is proper and injective. Whence $q \notin \varphi(\partial \mathcal{U})$ and $\varphi(\partial \mathcal{U})$ is closed in $N$. Let $\mathcal{V}$ be a connected component of $N \backslash \varphi(\partial \mathcal{U})$ containing $q$ which is its open neighborhood. Since $\mathcal{U}$ is connected, it folows that $\varphi(\mathcal{U}) \subset \mathcal{V}$. Then we get from $\varphi(\partial \mathcal{U}) \cap \mathcal{V}=\varnothing$ that $\overline{\mathcal{U}} \cap \varphi^{-1}(\mathcal{V})=\mathcal{U}$ and so $\left.\varphi\right|_{\mathcal{U}}: \mathcal{U} \rightarrow N$ is proper and injective. By Theorem 3.7 there is a point $x \in M$ such that the tangent map $T_{x} \varphi$ is injective and since $\operatorname{Ind} \varphi=0$ it is surjective too. Therefore, $y=\varphi(x)$ is a regular value with $\varphi^{-1}(y)=\{x\}$ and so $\operatorname{deg} \varphi=1$. It follows that $\varphi$ is surjective, because otherwise any point from $N \backslash \varphi(M)$ is regular and thus $\operatorname{deg} \varphi=0$ which is contradiction. Hence $\mathcal{V}=\varphi(\mathcal{U})$ is an open neighborhood of $q$.

An analogue of this theorem for Banach manifolds can be found in [11].

Corollary 3.9 (Nonlinear Fredholm alternative). Let $\varphi: M \rightarrow N$ be an $M C^{k}$-Lipschitz-Fredholm mapping of index zero, $k>1$. If $N$ is connected and $\varphi$ is locally injective, then $\varphi$ is surjective and finite covering mapping. If $M$ is connected and $N$ is simply connected, then $\varphi$ is a homeomorphism.

Proof. The proof is standard.

The following theorem is a generalization of the Borsuk-Ulam theorem. Its proof is a slight modification of the Banach case, cf. [3]. 
Theorem 3.10. Let $\varphi: \overline{\mathcal{U}} \rightarrow F$ be a non-constant closed LipschitzFredholom mapping of class $M C^{2}$ with index zero, where $U \underline{\underline{\Xi}} F$ is symmetric. If $\varphi$ is odd and for $u_{0} \in \bar{U}$ we have $u_{0} \notin \varphi(\partial \overline{\mathcal{U}})$. Then $\operatorname{deg}\left(\varphi, u_{0}\right) \equiv 1$ $\bmod 2$.

Proof. Since $\mathrm{D} \varphi\left(u_{0}\right)$ is a Lipschitz-Fredholm mapping with index zero

$$
F=\mathbf{F}_{\mathbf{1}} \oplus \operatorname{ker} \varphi=\mathbf{F}_{\mathbf{2}} \oplus \operatorname{Img} \varphi
$$

and $\operatorname{dim} \mathbf{F}_{\mathbf{2}}=\operatorname{dim} \operatorname{ker} \varphi$. The image $\varphi(\partial \mathcal{U})$ is closed as $\varphi$ is closed, hence

$$
\mathbf{a}=\varrho\left(\varphi(\mathcal{U}), u_{0}\right)>0
$$

because $u_{0} \notin \varphi(\partial \mathcal{U})$.

Let $\phi: F \rightarrow F$ be a global Lipschitz-compact linear operator with $\operatorname{Lip}(\phi)<\mathbf{b}$ for some $\mathbf{b}>0$. Define the mapping $\Phi_{\phi}: \bar{U} \rightarrow F$ by $\Phi_{\phi}(u)=\varphi(u)+\phi(u)$. Then $\Phi_{\phi}$ is a Lipschitz-Fredholm mapping of index zero. Suppose $\mathbf{b}<\mathbf{a} / \mathbf{k}$ for some $\mathbf{k}>1$, then

$$
\varrho\left(\Phi_{\phi}(u), u_{0}\right) \geqslant \varrho\left(\varphi(e), u_{0}\right)-\mathcal{L} i p(\phi) \varrho\left(U, u_{0}\right)>\mathbf{a}-\mathbf{b k}>0, \quad \forall u \in \partial \mathcal{U} .
$$

Therefore, $u_{0} \notin \Phi_{\phi}(\partial \mathcal{U})$. Hence $\operatorname{deg}\left(\varphi, u_{0}\right)=\operatorname{deg}\left(\Phi_{\phi}, u_{0}\right)$ as the mapping

$$
\psi:[0,1] \times \bar{U} \rightarrow F, \quad \psi(t, u)=\varphi(u)+t \phi(u),
$$

is proper and $u_{0} \notin \psi(\partial U)$ for all $t$. Considering the fact that

$$
\psi(-u)=-\psi(u)
$$

we may use the perturbation by compact operators to find the degree of $\varphi$. Let $\mathrm{C}$ be the set of global Lipschitz-compact linear operators $\phi: F \rightarrow F$ with $\operatorname{Lip}(\phi)<\mathbf{b}<\mathbf{a} / \mathbf{k}$. Let also $\hat{\phi} \in \mathbf{C}$ be such that its restriction to $\mathbf{F}_{\mathbf{1}}$ equals $u_{0}$ and

$$
\left.\widehat{\phi}\right|_{\operatorname{ker} \mathrm{D} \varphi\left(u_{0}\right)}: \operatorname{ker} \mathrm{D} \varphi\left(u_{0}\right) \rightarrow \mathbf{F}_{\mathbf{2}}
$$

is an $M C^{1}$-isomorphism. Then $\mathrm{D} \varphi\left(u_{0}\right)+\widehat{\phi}$ and consequently $\mathrm{D} \varphi\left(u_{0}\right)$ is an $M C^{1}$-isomorphism. Now define the mapping

$$
\Psi: \mathcal{U} \times \mathrm{C} \rightarrow F, \quad \Psi(u, \phi)=\Phi_{\phi}(u) .
$$

Then for a sufficiently small $\mathbf{b}$ the differential

$$
\mathrm{D} \Psi(u, \phi)(v, \psi)=(\mathrm{D} \varphi(u)+\phi) v+\psi(u)
$$

is surjective at $u_{0}$ as $\mathrm{D} \varphi\left(u_{0}\right)$ is an $M C^{1}$-isomorphism. It is also clear that it is surjective at the other points. Then, the mapping $\Psi$ satisfies the assumption of Theorem 2.6. Therefore, $\Psi^{-1}\left(u_{0}\right)$ is a submanifold and the mapping $\Pi: \Psi^{-1}\left(u_{0}\right) \rightarrow$ C induced by the projection onto the second order is Lipschitz-Fredholm of index zero. By employing the local version of Sard's theorem we may find a regular point $\bar{\phi}$ of $\Pi$, and from the proof of the Theorem 2.6 it follows that $u_{0}$ is a regular value of $\Phi_{\bar{\phi}}$. Hence $u_{0}$ is a 
regular value of $\varphi$. Thus, the properness and the identity $\varphi(-u)=-\varphi(u)$ imply that

$$
\varphi^{-1}\left(u_{0}\right)=\left\{u_{0}, f_{1},-f_{1}, \cdots f_{m},-f_{m}\right\}
$$

and therefore $\operatorname{deg}\left(\varphi, u_{0}\right) \equiv 1 \bmod 2$.

\section{REFERENCES}

[1] Kaveh Eftekharinasab. Sard's theorem for mappings between Fréchet manifolds. Ukr. Math. J., 62(12):1896-1905, 2011.

[2] Kaveh Eftekharinasab. Transversality and Lipschitz-Fredholm maps. Zb. Pr. Inst. Mat. NAN Ukr., 12(6):89-104, 2015.

[3] Kazimierz Gȩba, Andrzej Granas. A proof of the Borsuk antipodal theorem for Fredholm maps. J. Math. Anal. Appl., 96(1):196-202, 1983.

[4] Helge Glöckner. Implicit functions from topological vector spaces to fréchet spaces in the presence of metric estimates. https://arxiv.org/pdf/math/0612673.pdf, 2007.

[5] Helge Glöckner. Fundamentals of submersions and immersions between infinitedimensional manifolds. https://arxiv.org/abs/1502.05795, 2015.

[6] Gareth Jones, Jonathan Kirby, Olivier Le Gal, Tamara Servi. On local definability of holomorphic functions. The Quarterly J. Math., 70(4):1305-1326, 2019.

[7] Juan Margalef-Roig, Enrique Outerelo Dominguez. Differential Topology, volume 173 of North-Holland Mathematics Studies. North Holland, 1992.

[8] Olaf Müller. A metric approach to Fréchet geometry. Journal of Geometry and physics, 58(11):1477-1500, 2008.

[9] Ruslan Sadyrkhanov. On infinite dimensional features of proper and closed mappings. Proceedings of the AMS, 98(4):643-648, 1986.

[10] Steve Smale. An infinite dimensional version of Sard's theorem. Amr. J. Math, 87(4):861-866, 1965.

[11] Anthony Tromba. Some theorems on Fredholm maps. Proceedings of the AMS, 34(2):578-585, 1972.

Received: January 21, 2021, accepted: June 21, 2021.

Eftekharinasab Kaveh

Topology lab. Institute of Mathematics of National Academy of Sciences of Ukraine, Tereshchenkivska st. 3, Kyiv, 01601 Ukraine

Email: kaveh@imath.kiev.ua

ORCID: 0000-0002-4604-3220 